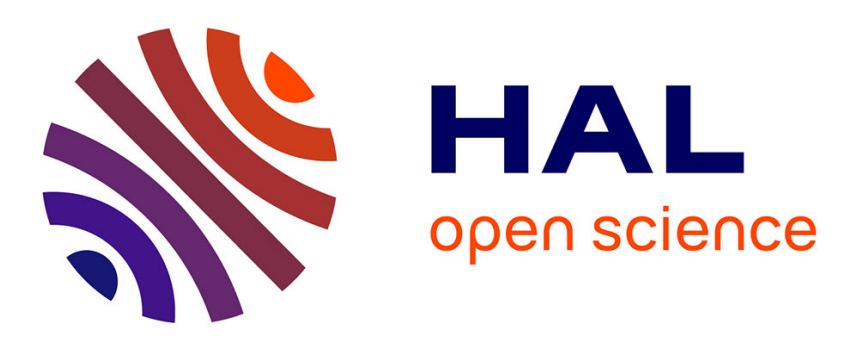

\title{
Alignment of the product lifecycle management federated interoperability framework with internet of things and virtual manufacturing
}

David Tchoffa, Nicolas Figay, Parisa Ghodous, Hervé Panetto, Abderrahman El Mhamedi

\section{To cite this version:}

David Tchoffa, Nicolas Figay, Parisa Ghodous, Hervé Panetto, Abderrahman El Mhamedi. Alignment of the product lifecycle management federated interoperability framework with internet of things and virtual manufacturing. Computers in Industry, 2021, 130 (103466), 10.1016/j.compind.2021.103466 . hal-03214734

\author{
HAL Id: hal-03214734 \\ https://hal.science/hal-03214734
}

Submitted on 2 May 2021

HAL is a multi-disciplinary open access archive for the deposit and dissemination of scientific research documents, whether they are published or not. The documents may come from teaching and research institutions in France or abroad, or from public or private research centers.
L'archive ouverte pluridisciplinaire HAL, est destinée au dépôt et à la diffusion de documents scientifiques de niveau recherche, publiés ou non, émanant des établissements d'enseignement et de recherche français ou étrangers, des laboratoires publics ou privés. 


\title{
Alignment of the Product Lifecycle Management Federated Interoperability Framework with Internet of Things and Virtual Manufacturing
}

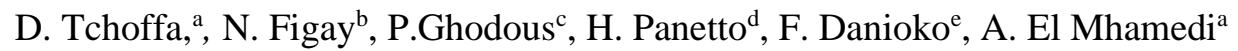 \\ ${ }^{a}$ Quartz laboratory (EA 7393), Paris 8 University, Montreuil, France (e-mail: \\ \{d.tchoffa\} \{a.elmhamedi\}@iut.univ-paris8.fr \\ b Airbus, Suresnes, France (e-mail : nicolas.figay@gmail.com) \\ ${ }^{c}$ LIRIS, Lyon, France (e-mail : parisa.ghodous@univ-lyon1.fr) \\ d Université de Lorraine, CNRS, CRAN, Nancy, France (e-mail : herve.Panetto@univ-lorraine.fr )
}

\begin{abstract}
The emergence of the Internet of Things (IoT), coupled with other capabilities such as Cloud Computing and many others, creates opportunities for the development of Cyber-Physical Systems (CPS) at the scale of a digital business ecosystem. It will impact the way engineers work when designing, developing, deploying, operating, or maintaining complex systems-of-systems, with increasing needs concerning security, agility, and interoperability. The Federated Interoperability Framework (FIF) has been developed for Aeronautic Space and Defence over the last years to prepare and build continuous operational Product Lifecycle Management (PLM) interoperability. This paper aims at illustrating, defining, and demonstrating the required evolutionary nature of the FIF from its genesis, by describing past, last, and future evolutions, in particular for the PLM Standards testbed, Virtual manufacturing and CPS. Then Evolvability is defined and discussed, and the provided presentation of the FIF evolutions used to demonstrate its evolutionary nature, making the FIF contributing to the State of the Art and the State of the Practice.

Keywords: Internet of Things, Product life cycle management, Virtual enterprises, Virtual Manufacturing
\end{abstract}

\section{I.INTRODUCTION}

Internet evolution, with the emerging Internet of Things (IoT), Cloud Computing and many others, creates opportunities for the development of a new kind of digital business ecosystem constituting Cyber-Physical Systems (CPS) at scale. It also supports new trends and evolutions which will define the Product Lifecycle Management (PLM) [1] of the future, i.e. the adaptation of PLM approach and solutions for encompassing Manufacturing Industry's trends such as Digital Twins or Circular Economy. A Digital Twin is a concept describing a collection of digital data representing a physical object, having its roots in engineering and the creation of engineering drawings/graphics. It is a new kind of digital artifact aiming at the continuous improvement in the creation of product design and engineering activities. It is closely related to Virtual Manufacturing and predictive maintenance. Digital twins have consequently to be managed in configuration and exchanged or shared between various actors according to the PLM processes, using new dedicated PLM standards (e.g. [QIF]). The Circular Economy idea is to avoid the waste of not inexhaustible resources related to human activities and leading to their rarefaction and to the destruction of the natural ecosystem. It will be done by supporting the recycling of products that will consequently have several lives, through localized services closer to users. New regulations will also probably emerge. New emerging actors' activities around the product will have to be considered by PLM processes. New associated standards or new versions of standards will have to be considered for new digital collaboration needs, while maintaining the operational interoperability during enterprises' transformation and continuous refactoring of the information system, without preventing the ability of exchanging, sharing and archiving the legacy data which shall be preserved despite the changes.

The way engineers work and PLM will be impacted by these evolutions when designing, developing, deploying, operating, and maintaining complex Systems of Systems (SoS) (e.g., Aircraft and associated design, production, operating and support environment). In particular, the complexity of the SoS should be considered and the need for continuous secured communications and interactions of several systems independently will increase, making the required interoperability an actual challenge.

To prepare and build the required continuous operational interoperability for PLM at an acceptable cost, the Federated Interoperability Framework (FIF) has been developed during the last twenty years. It was designed for the adaptation to the continuously changing business and technical environments, such as those related to the previously described trends, for supporting Product and Process Data Exchange, Sharing and Long Term Archiving required in Industrial Domains where the duration of life of a Product is longer (more than 50 years) than the ones of the used Information and Communication technologies (ICT) and related standards, with smaller durations of life. E.g. in 30 years, we faced the rising and decline of several middleware 
technological framework for software product interoperability such as [CORBA], the Web services oriented framework for the support of the eBusiness relying on XML technologies, and today new architectural microservices frameworks based on Cloud and JavaScript technologies. This paper aims at demonstrating the FIF evolutionary nature, e.g. its ability to be adapted to the high pace of change of business and ICT environments, while maintaining continuously the interoperability of PLM applications at an acceptable price.

For the PLM community, open standards are key enablers for preparing and building the operational interoperability required for PLM. Several are mentioned in the paper, which were assessed and elected as building blocks of the collaborative platform built using the FIF. It includes 1) Manufacturing Data Standards application protocols for exchanging, sharing and archiving data describing the products, with the associated processes and production systems or support systems 2) Enterprise and System modeling languages 3) ICT standards for the support of data exchange for the support of distributed interacting software solutions (also called middleware) or for the support of business process modeling and automation. 4) Virtual Manufacturing or Industrial Internet 6) Multi syntax languages for information models or ontology 5) Enterprise modeling. Some of those which have been considered by the FIF related research are listed in the table with their successive versions (years). If some are International Standard Organization (ISO)'s standards, others are specifications coming from various consortia: Object Management Group (OMG), Open Group, Organization for the Advancement of Structured Information Standards (OASIS), International Electro technical Commission (IEC), and American National Standards Institute (ANSI). Some competition exists between these organizations. As a consequence, important overlaps, incompatibility but also complex interdependencies have to be managed when willing to cover the whole spectrum of functional needs, business constraints related to their mandatory usage but also interoperability.

[STEP] ISO 10303 STandard for the Exchange of Product model data https://en.wikipedia.org/wiki/lSO 10303

[AP203] ISO 10303-203:(1994, 2005, 2011) : Configuration controlled 3D designs of mechanical parts and assemblies [https://www.iso.org/fr/standard/44305.html]

[AP214] ISO 10303-214:(2001, 2003, 2010) : Core data for automotive mechanical design processes[https://www.iso.org/standard/43669.html] [AP242] ISO 10303-242:(2014, 2020): Configuration controlled 3D designs of mechanical part and assemblies[https://www.iso.org/standard/66654.html]

[STEP NC] AP238 2007: Application interpreted model for computerized numerical controllers [AP238] AP238 2020: Model based integrated manufacturing

[PDM Enablers] OMG's PDM Enablers v1.3, November 2000 [https://www.omg.org/spec/PDME/1.3/PDF]

[ISA 95] ISO/IEC 62264 - Enterprise-Control System Integration [https://www.iso.org/standard/57308.html], the international version of ISA 95

[PLCS] OASIS Product Life Cycle Support https://www.oasis-open.org/committees/tc_home.php?wg_abbrev=plcs

[MDA] OMG Model Driven Architecture http://www.omg.org/mda/

[CORBA] OMG Common Object Request Broker Architecture https://corba.org/

[OMA] OMG Object Management Architecture https://www.omg.org/gettingstarted/specintro.htm\#OMA

[OSLC] OASIS Open Services for Lifecycle Collaboration https://www.oasis-open.org/committees/tc home.php?wg abbrev=oslc-domains [OPC UA] OPC Foundation Open Platform Communication Unified Architecture https://opcfoundation.org/about/opc-technologies/opc-ua/ [QIF] ISO 23952:2020 Quality Information Framework https://www.iso.org/fr/standard/77461.html

[RAMI] IEC PAS 63088:2017 -Smart manufacturing - Reference architecture model industry 4.0 https://webstore.iec.ch/publication/30082

[PERA] Purdue Enterprise Reference Architecture https://en.wikipedia.org/wiki/Purdue_Enterprise_Reference_Architecture

[NIST Reference Model] Current Standards Landscape for Smart Manufacturing Systems http://dx.doi.org/10.6028/NIST.IR.8107

[MT Connect] ANSI/MTC1.4-2018 MT Connect https://www.mtconnect.org/

[ArchiMate] Open Group ArchiMate 3.1 https://pubs.opengroup.org/architecture/archimate3-doc/

[OWL] W3C Ontology Web Language 2 https://www.w3.org/TR/owl2-overview/

[PDM Enablers] Product Data Management Enablers Version 1.3, OMG, November 2000, https://www.omg.org/spec/PDME/1.3/

Table 1: Open standards, models and frameworks considered by the FIF

The demonstration is based on the study of the different successive projects which contributed to the development of the FIF and of the related research papers which contributed to the state of the art for PLM interoperability.

The Section II studies the FIF origins, purpose and evolutions over 20 years for assessing and federating several successive technological frameworks, Computer Aided Engineering practices and PLM standards.

The Section III presents the last evolution defined by a FIF related research project, addressing interoperability testing.

The Section IV presents future planned extensions for Virtual Manufacturing, Cyber Physical Systems or Autonomous Systems. It demonstrates that FIF which was defined and used in the last 20 years can be used also for preparing and building the Interoperability for the PLM of the future.

The section V discusses the required evolutionary nature of the FIF for interoperability, comparing it with other legacy or emerging interoperability frameworks.

\section{THE FEDERATED INTEROPERABILITY FRAMEWORK AND ITS FIRST EVOLUTIONS}

The Federated Interoperability Framework [2] has been developed in recent years to establish sustainable interoperability of 
product and process data. It was carried out by Airbus Group Innovation (AGI) through successive research and operational projects. It has been driven by the development and use of manufacturing data standards as catalysts for PLM interoperability within the Aeronautical, Defence and Space digital business ecosystem. The FIF is based on the combined use of open standards in the Manufacturing, Information Systems (IS) and Information \& Communication Technologies (ICT) fields. It aims to prepare and build the required continuous operational PLM interoperability at an acceptable price in constantly evolving business and technical environments. Its development was motivated by and performed through the participation in many research projects followed by the assessment in operations of the results of these research projects. It led to the development of an experimental collaborative platform associated with the FIF, the cPlatform. Figure 1 represents the timeline of the FIF Development with related research projects and successive versions of the cPlatform. The FIF development started with a PhD research works on Interoperability of Technical Enterprise Applications [2] («Thesis Inter» on Figure 1). The last evolution (cPlatform v4) is an outcome of the SIP project [3]. Various studies not associated with a research project allowed to assess the required evolution for Virtual Manufacturing and Cyber-Physical Systems (Ass. VM/CPS) but also the opportunities related to emerging technologies and trends.

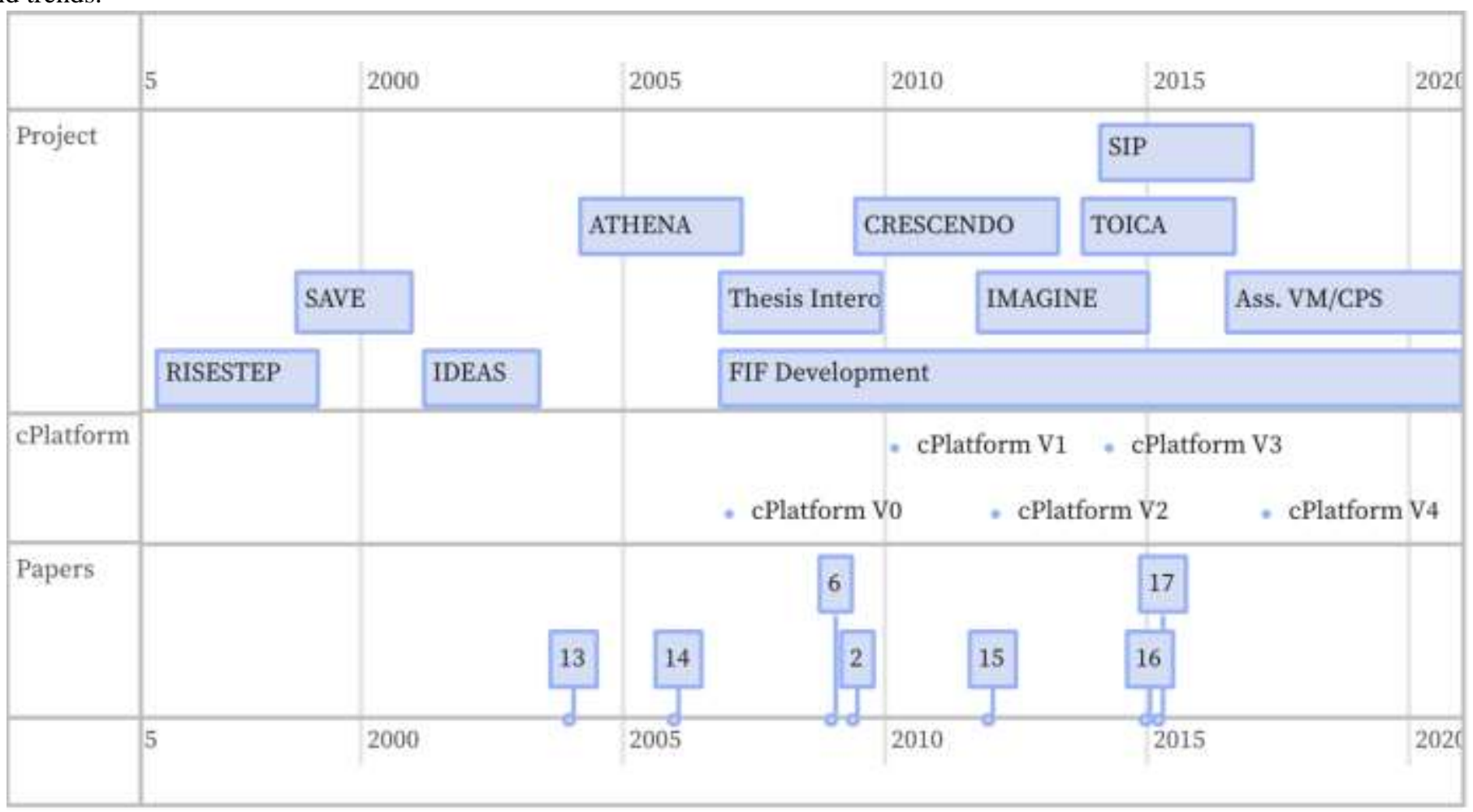

Figure 1: Timeline with FIF development, contributing projects and cPlatform evolutions over 20 years

At the very beginning, the two European Research Projects RISESTEP (FP4 ESPRIT 20459 - Enterprise Wide Standard Access to STEP Distributed Databases) and SAVE ( FP4 BRITE/EURAM 3 BRPR 980760 - STEP In a Virtual Enterprise) aimed at and succeeded in developing a middle-ware environment dedicated to Digital Mock-Up unit sharing within a distributed heterogeneous environment. They were based on STEP for information models and on CORBA for middleware.

Despite the demonstrated feasibility of the proposed approach and the produced open standard ([PDM Enablers]), the results were not adopted by industry and the developed standard didn't become a de facto standard.

According to [2], different AGI studies identified the reasons preventing their usage and deployment in the enterprise for establishing the required interoperability. One of them - the disconnection of the Manufacturing data standardization community and Enterprise Information system architects' community - led to the launching of two European Research projects: IDEAS (EC FP5 IST-2001-37368 Interoperability Development for Enterprise Application and Software) research roadmap project and ATHENA (EC FP6 IST 507849 Advanced Technologies for Interoperability of Heterogeneous Enterprise Networks and their Application). The proposed business case was the PLM collaboration based on open manufacturing standards with the associated lessons learnt from the encountered issues from RISESTEP and SAVE results.

For IDEAS and ATHENA, interoperability, defined as "the ability of two or more systems or components to exchange information and to use the information that has been exchanged", was addressed in respect of all layers of an enterprise. It was tackled using a holistic perspective, a multi-disciplinary approach, and by bringing together the best research teams, industry expertise and ICT suppliers. The goal was to remove barriers to interoperability, to transfer and apply the research results in industrial sectors, and to foster a new networked business culture. However, ATHENA failed to provide usable results [4] [5]. 
[2] provides the following reasons: 1) Unlike the manufacturing community involved in RISESTEP and SAVE, the ATHENA partners defined their Integration Framework after launching the different ATHENA sub projects dedicated to the production of component solutions related to the different identified enablers: Model based approach, Ontology, Service Oriented Architecture and Enterprise Modeling. As a result, it was impossible for the Industrial partners to perform the integration of these solutions and to build the expected interoperability. 2) Researchers and solution providers in ATHENA did not rely on the usage of manufacturing standards as requested by Industrial partners. 3) Accessing and consequently using the results of these projects after their completion was impossible, as the public web sites were closed a few months after the end of the projects and consequently public results were not available anymore. 4) Many results which should have contributed to the interoperability of enterprise applications within the supply chain were confidential and not industrialized. 5) The developed demonstrators produced by software vendor partners for each project were not made available for the next projects. A lot of resources were wasted for redeveloping already existing functional components, in place of concentrating on new innovative components with high added value 6) Silos between operations and research were also identified as an interoperability brake (i.e. something preventing setting up interoperability), as well as disruptive research without consideration on the innovation path allowing sustainable interoperability. 7) PLM interoperability research is too much restricted to the perimeters of the different functions of the enterprises, i.e., Design, Production or Support. It prevents the Through Lifecycle Interoperability creating silos and sets of inconsistent standards in the Supply Chains of a Digital Business Ecosystem.

What was first learnt from these four projects is that Openness is required for interoperability. Indeed, producing an open experimental modular collaborative platform which can be reused and extended over the time and the projects is also needed. Another issue is the pace of change of Information and Communication Technologies which is continuously growing. E.g. [CORBA] studied in SAVE has been replaced by Web services relying on XML standards in ATHENA, themselves replaced by Micro Services and JSON standards today. [AP203] in RISESTEP was replaced by [AP214] in SAVE, replaced today today by [AP242]. However, the considered manufacturing data should be used on a longer period, exceeding the one when a technology is deployed, used, and then decommissioned. Therefore, the change of technologies is disruptive. It breaks and limits the establishment of interoperability by creating many technological silos. Open standards, due to their longer life duration, should contribute to a smoother and mastered transition when organizational or technological changes occur. Consequently evolvability is a required quality of any PLM Interoperability Framework.

The successive failures and their analysis led to the launch of a PhD research work [2] at the origin of the FIF, aiming at taking advantage of the lessons learnt. The FIF approach consists first in characterizing an ideal collaborative system according to legacy relevant interoperability frameworks. It consists secondly in applying principles, and using enablers, defined by the ATHENA interoperability framework (AIF) [5]. However, the FIF extends the AIF by identifying interoperability breakthroughs to be unleashed [2] and complementary interoperability enablers to be used, in particular open standards and open-source software in a combination which is considered as strategic for an evolutionary framework [2], having to consider a longer term than the ones associated with applications or technologies. Indeed, the availability of industrial quality open source software solutions implementing open standards is mandatory [6] for assessing maturity and usability of these standards and establishing sustainable PLM interoperability. They are also the key for building an experimental collaboration platform for assessing these standards and their combination, including continuously new technologies and reconfiguring the components for experimenting new approaches. Standardization governance, with its associated interoperability framework of reference, is also required when assessing the interoperability maturity level [7] of a business community.

Consequently, the FIF continuous development and evolution adopted a strategic long-term approach, with iterations between research, operational and standardization projects. Through efficient dissemination and exploitation, the FIF contributed to the development of Aeronautic, Space and Defence (ASD) digital community in Europe, contributing to the development of its maturity in terms of interoperability. Links between ASD Strategic Standardization Group (SSG) have been established with many other communities contributing to PLM interoperability: ISO Technical Committee for Manufacturing Data, the European research cluster for Enterprise Applications Interoperability, etc. It allowed alignment between research, standardization ([AP242], [STEP] updated Architecture) and operational projects (Airbus PLM harmonization, Boost Aerospace PLM Hub)

The FIF development also contributed to the State of the Art, by proposing 1) The Extended Hypermodel for Interoperability (EH4I), a semantic hypergraph capturing, for each business concept, the equivalent construct for each language in use during conceptualization, design, development, and execution phases of an application. Such a multiple representation allows semantic preservation and prevents data loss when performing PLM data exchange or sharing 2) The Dynamic Manufacturing Networks (DMN) concept is the last FIF evolution before SIP and developed during IMAGINE [8]: a network of enterprises working on a same manufactured product all along its life cycle, with the associated internal private processes and external cross organizational processes, the applications supporting (serving or being used by) these processes and the distributed software systems realizing them. The figure 2 gives a simplified example of a DMN related to an Airbus product, restricted to enterprises and processes. A DMN should be modeled using an open standardized Enterprise modeling language. The relevance of using an open neutral standard to interconnect two applications requires applying this approach at scale within such a DMN. A holistic approach implied by enterprise architecture is not sufficient, as it is restricted to a single enterprise. In a mature community in terms of interoperability, a neutral standard is appearing as a network protocol, enabling interoperability at scale, and creating the actual 
value by the savings it generates.[9][10][11] are publications about DMNs, modeled with ArchiMate 2. .

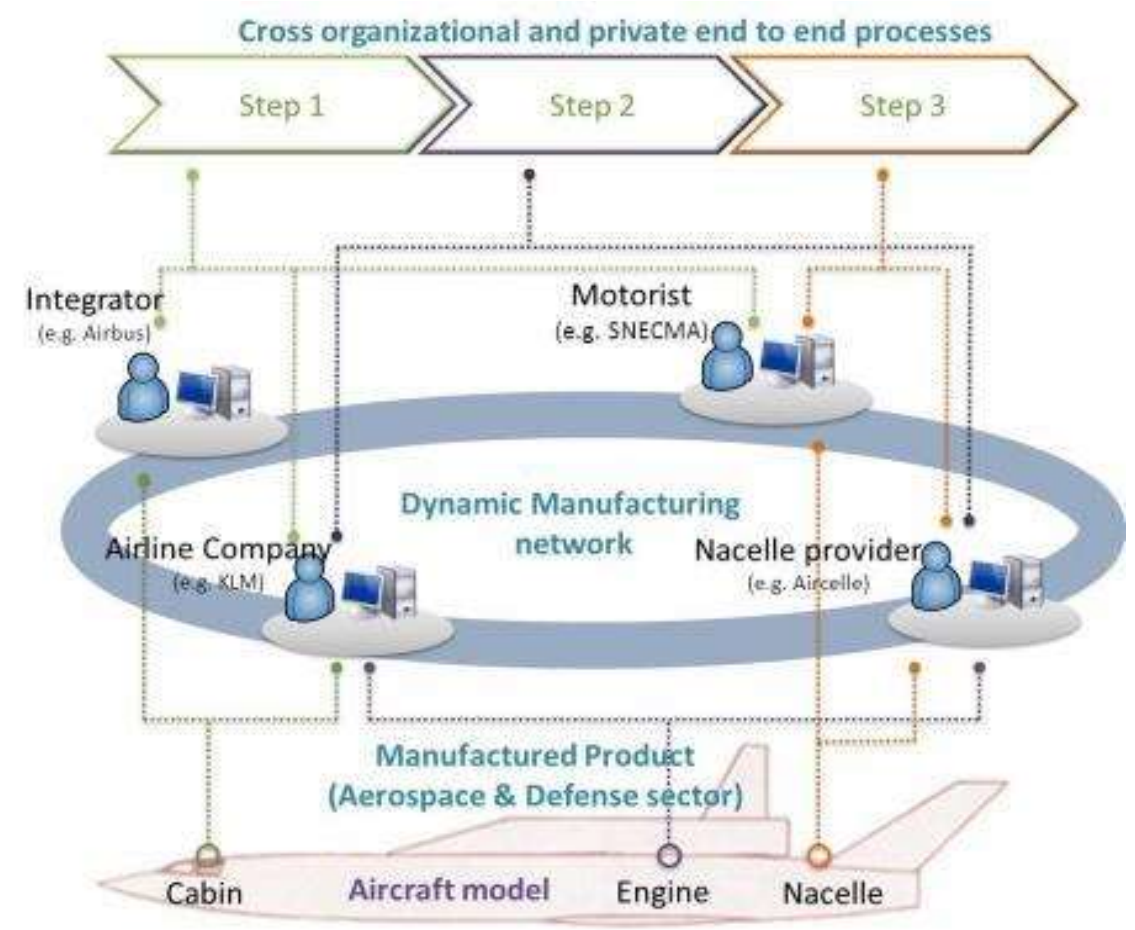

Figure 2: Illustration of e Dynamic Manufacturing Network through an example

As a conclusion, the FIF had been defined and used by successive research projects over a period of 20 years with three successive versions of the cPlatform, and actual results on standardization and industry. How it contributed to the state of the art over this period is reflected in table 2. The next section describes the last realized evolution of the FIF and of the associated cPlatform, aiming at unleashing the interoperability brake related to PLM Standards assessment and interoperability testing within a DMN. It also illustrates a more concrete way the approach proposed by the FIF and underlying model generated cPlatform.

\begin{tabular}{|c|c|c|}
\hline FIF related paper & Year & Contribution to the FIF \\
\hline [13] & 2004 & $\begin{array}{l}\text { Extended Hypermodel for Interoperability (EH4I) is proposed for preventing data loss during transformations used for } \\
\text { model based interoperable application generation, from conceptual to design model, and from model to application. }\end{array}$ \\
\hline [14] & 2006 & Knowledge models in [OWL] or [UML] can be generated from STEP APs for building interoperability of application \\
\hline [6] & 2009 & Importance of Open Source is demonstrated when having to deal with interoperability and related standards \\
\hline$[2]$ & 2009 & $\begin{array}{l}\text { This thesis defines the foundation of the FIF. Combining usage of legacy interoperability models, business standards and } \\
\text { technological framework, it defines the interoperability of technical enterprise applications, and proposes a pragmatic way } \\
\text { for preparing and building continuous operational interoperability at an acceptable cost. It comes with a pragmatic approach } \\
\text { for identifying both relevant enablers and brakes -i.e. what prevents the establishment of interoperability and has been } \\
\text { identified from assessment of current practices and approach - in order to solve them appropriately through business, } \\
\text { standardization or research activities. The approach has been applied during } 12 \text { years, reflected in this paper. }\end{array}$ \\
\hline [15] & 2012 & $\begin{array}{l}\text { The FIF is enriched for addressing collaboration within Dynamic Manufacturing Network, extending the set of covered } \\
\text { PLM standards with [ISA95] and relying on Blueprint templates formalized with ArchiMate. }\end{array}$ \\
\hline [12] & 2014 & $\begin{array}{l}\text { Dynamic Manufacturing Network, PLM Hub and Business standards testbed. } \\
\text { This approach is completed by the collaboration of an associated testbed for eBusiness PLM standards allowing the } \\
\text { community to accelerate development of required standards as well as their implementation } 2014\end{array}$ \\
\hline [16] & 2015 & $\begin{array}{l}\text { The process for producing DMN blueprint template is detailed, extending its usage to PLM standards assessment and to } \\
\text { the conformance testing of implementations of these standards. }\end{array}$ \\
\hline [11][17] & 2015 & Assessment of [ISA95] for usage on a concrete manufacturing case relying on the FIF \\
\hline
\end{tabular}

Table 2: FIF first iterations related publications 


\section{The LAST EVOLUTION RELATED To INTEROPERABILITY TESTING}

The last evolution of the FIF is described in this section and its evolutionary nature demonstrated for being able to address testability identified brakes, from the Return on EXperience (REX) of IMAGINE and PLM harmonization at Airbus. SIP proposed approach should allow assessing standards and their implementations on top of an experimental test bed platform simulating a complex DMN at an acceptable price, extending the FIF with new capabilities. For this, introducing virtualization and cloud technologies was identified as an interoperability enabler, while SIP was the opportunity to integrate Cloud technologies in the collaborative platform and to take advantage of it for simulating complex multi-partners digital and secured collaboration at scale.

States of the Practice (SoP) and States of the Art (SoA) performed in [9] and [10] show that no approach addressing the identified needs and solving the identified scientific gap was found. When performed, no formal method was existing that allows modeling DMN interoperability use cases, derived collaboration models and associated testing procedures with the needed test data. As many proposed testbed approaches, [18] only deal with schemas conformance, which is not interoperability testing, within a B2B context. [19] proposes exactly what should be considered as interoperability testing but restricted only to the ICT layer. The adopted philosophy was [19] similar, but considering the more holistic FIF multi-layered approach, the manufacturing context and the enabling virtualization cloud technologies in order to shorten the time to usage of PLM manufacturing standards. For that, a DMN Software Factory (DSF) was proposed which takes advantage of a Model Driven Approach for the fast generation and assembly of testing environment components in the Cloud. The FIF, the building blocks of testing environments of the DSF should be extensible and reconfigurable to support evolution of technologies and practices for continuous interoperability.

In terms of Business case, the starting situation is the one illustrated by the figure 3. A cross organizational collaborative process $(\mathrm{Pc})$ is to be set up to realize a Business Collaboration between Original Equipment Manufacturer (OEM) and a Supplier. Each of them has a private process, served by an application, and realized by a software system, resulting from the deployment and parameterization of a software Product in an operational business and technical environment. Let us note that the used terminology is a usual one for Enterprise Architecture in general, and for ArchiMate in particular. A first principle of the FIF applied here and inherited from AIF is to consider that Interoperability is to be addressed at Business, Information System and Technology layers simultaneously. At business layer, it means that for two business processes, work requests and resulting outputs of the performed work are properly defined as inputs and outputs of the OEM and supplier's processes, intermediated by the cross organizational collaboration process. At the information system layer, it means that the relevant information must be shared between the applications supporting the processes for effective collaboration. Finally, information sharing will be realized concretely at the information and technology layer by exchange, sharing or linking data relying on various potential technology solutions.

From the AIF, the first version of the FIF proposed as an evolution the systematic usage of Manufacturing Data standards as a communication protocol between applications, on top of relevant technical solutions and open standards for communication between software systems. Relying on the STEP standard, applications and software products are categorized according to Conformance Classes (CC), which depend on sets of assigned (applications) or implemented (software products) Unit of Functionalities. The information flows to be implemented are the relevant ones for supporting the cross organizational processes. They are not requiring using all the concepts defined in an application protocol, but just the sufficient subset for covering the expected collaboration and for reflecting the distribution of functions to be performed between partners and application for a given product component. It is the reason why the Information Coverage concept was created, associated with each flow to implement between two applications. It is the subset of concepts in an application protocol needed for this flow. Information sharing needs being driven by collaboration to be supported, it was identified that implementation of the support by software vendors can be done progressively relying on a set of Standardized cross organizational collaborative processes (SPc) adopted by the members of a DMN or by a given digital business ecosystem mature in terms of interoperability. It can also be very useful for specifying formally the different business cases and derived use cases to be realized for collaboration.

Consequently, SIP considers that a collaboration process is to be associated with a Standardized Cross Organizational collaborative process (SPc) with an associated application protocol for which a Conformance Classes (CC) can be assigned to each application implied in the collaboration. Considering the figure 2, we will have respectively $\mathrm{CC}(\mathrm{O})$ and $\mathrm{CC}(\mathrm{S})$ the conformance class of respectively the application $\mathrm{O}$ and of the application $\mathrm{S}$. 


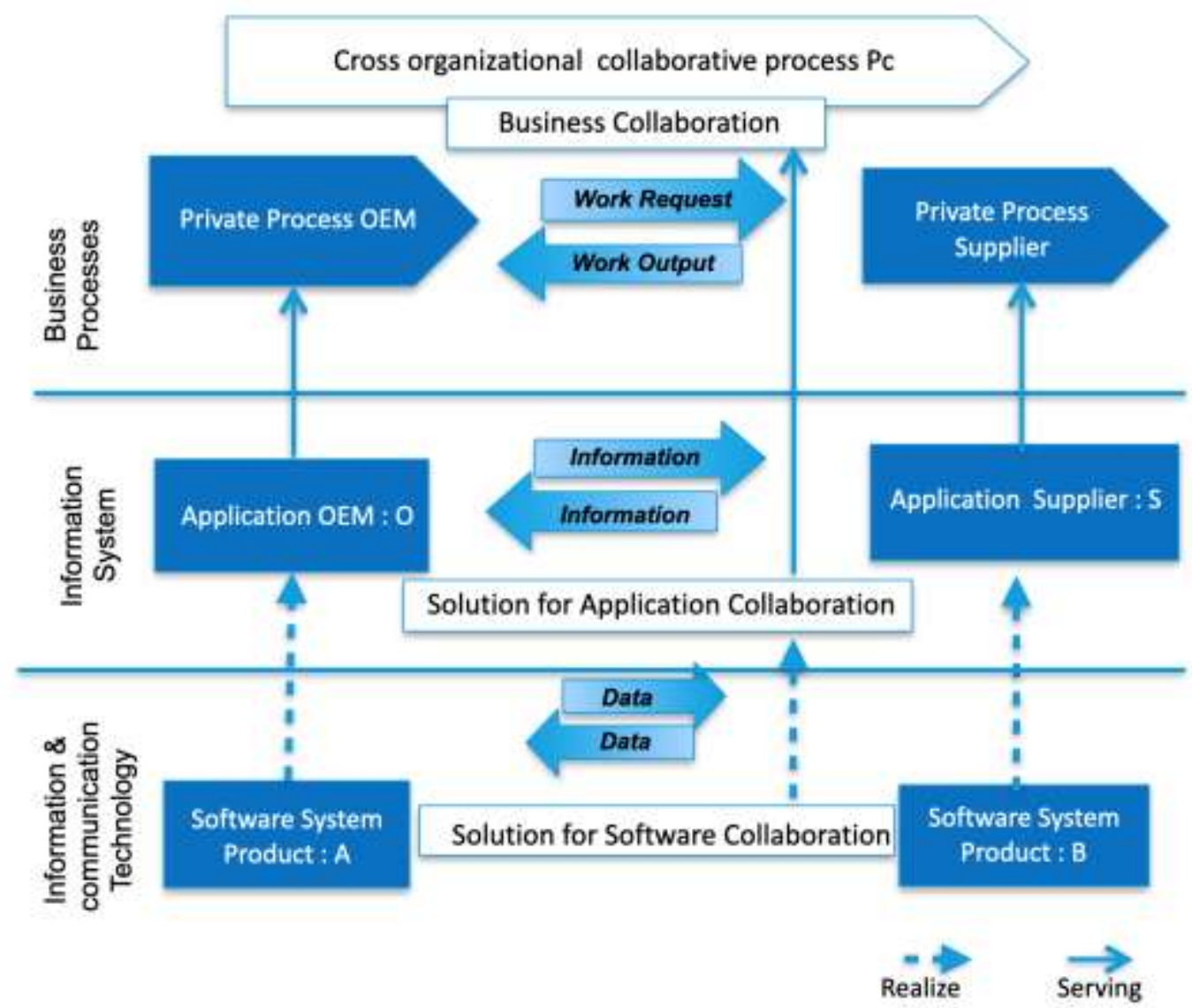

Figure 3: The three layer cross organizational collaboration to be implemented

SIP defines a 3 phases approach, for implementing a cross organizational collaboration within a DMN, relying on a generic [STEP] Application Protocol we name «APxx » on Figures 4 and 5 (with the associated reference model, schema and format) and a standard Cross Organizational collaborative process SPc. The first phase is the simulation phase (Cf. Figure 4), for checking alignment between the AP and the cross organizational collaborative process. At this phase, the goal is not to test the actual applications of OEM and Suppliers, but the adherence of the standard with Product \& Process data exchange needs. In light blue is the model that will be captured as an ArchiMate blueprint from which the testbed component will be deployed and parameterized. It's done manually first and automated with the DSF when qualified.

Doing so, a generic and shared portfolio of cross organization collaboration scenarios formalized as cross organizational collaborative Processes models $(\mathrm{Pc})$ is produced, from which can be derived the Standardized Cross Organizational collaborative processes (SPc) as business workflow models, data flows, associated test data sets and the required simulation and testing capabilities. The DMN model, formalized by means of ArchiMate, captures the actual data exchange environment distributed on ICT, Information System (IS) and Business Layer. It is used as the input of the DSF.

Such an approach was demonstrated in [9]. However, the collaboration environment is simulated on a multi-tenant network of virtual networks. The applications are also simulated with Application Reference Components (ARCs), applications with interfaces implementing the PLM standard subset required for playing the collaboration scenarios. It can be a legacy software product, as well as software components automatically generated from standards for playing the expected role. Such an approach was demonstrated in [20] and [21]. 


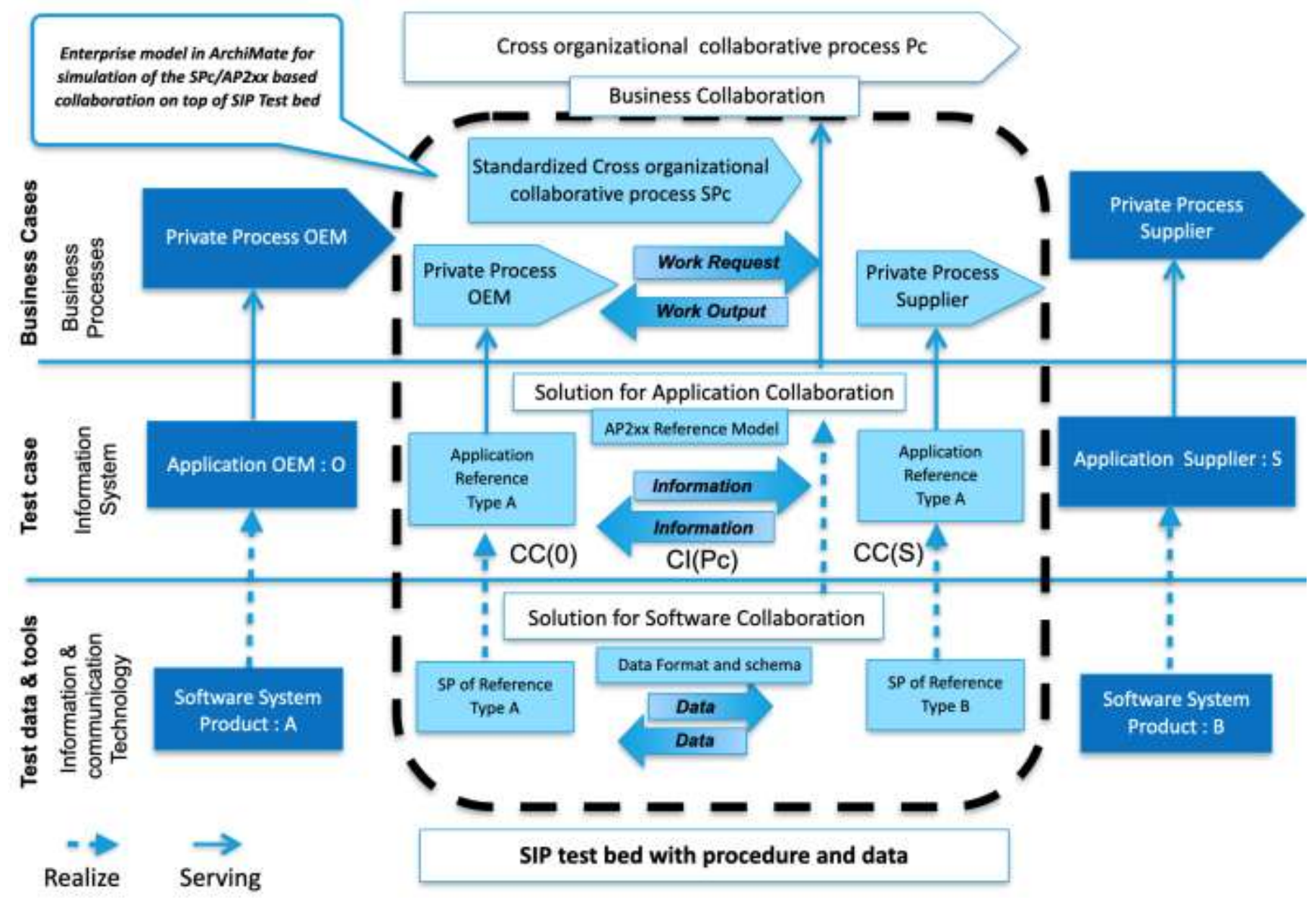

Figure 4: Modelling the collaboration based on PLM standards for their assessment

The second phase (cf. Figure 5) consists in testing software solutions or applications, within the role of ARCs used in the collaboration scenario. ARCs are replaced by applications or software systems to be tested. They can then be assessed as implementation of standards by replaying the testing scenarios developed in the first phase. It can be unitary testing (test of one application or solution), or integration testing (test of several applications playing different roles in the collaboration). It is the testing phase for standard implementation validation, which will result in a set of qualified applications supporting the targeted collaboration process through the usage of the PLM standards, used as DMN network protocols. Such an approach was demonstrated in [22]. Particularly appropriate places for such testing are legacy Implementer forums, such as the ones set up by AFNET, ProSTEP and NIST associations which are supporting development and usage of open standards by Industry. 


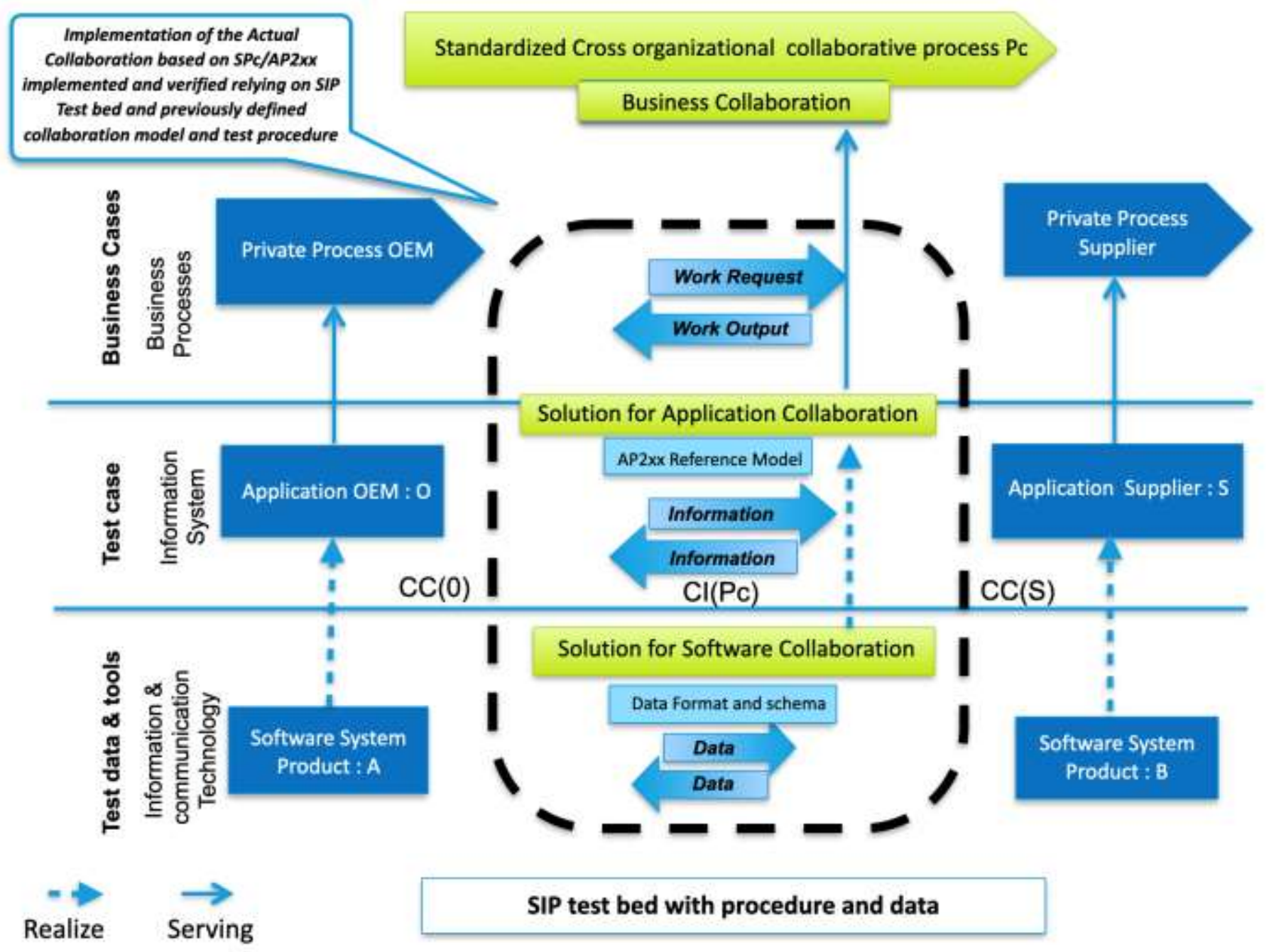

Figure 5: Testing and validating application Components as collaboration participants

The third phase (Cf. Figure 6) consists in monitoring interoperability of actual applications by tracking issues and being able to take actions when non-interoperability is detected, or to deal with interoperability maintenance when some changes must occur. In this phase, the test bed can be used as a monitoring solution to detect interoperability issues and to be able to address them very quickly. For the approach to be efficient, industrial partners, stakeholders, Information Management departments, Strategic Governance and Operations must be involved in the difference phases of the approach, as they are all playing an important role for defining the expected interoperability. 


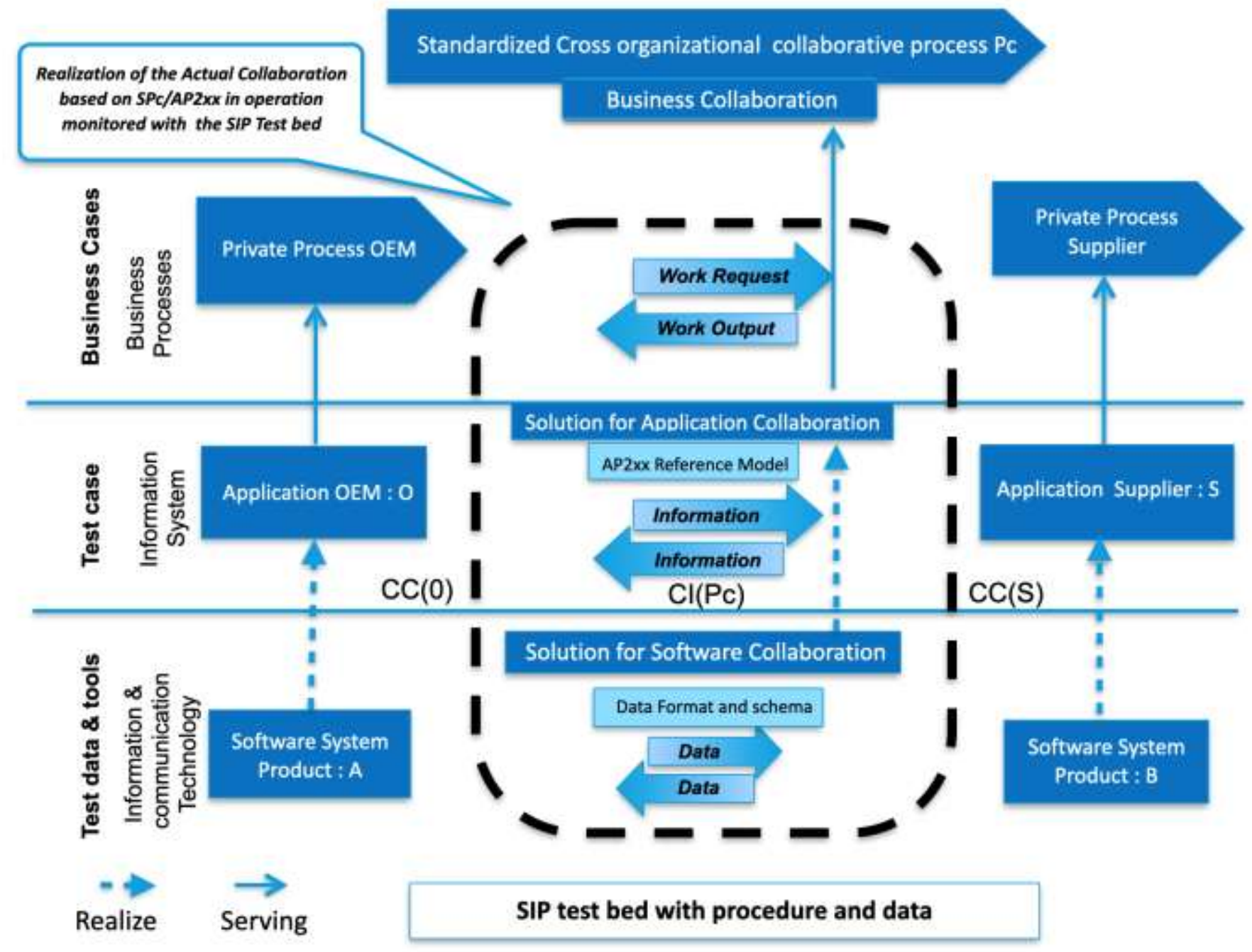

Figure 6: Monitoring interoperability in operation

Concerning the supporting platform, SIP simulation platform was derived from the last version of the collaborative platform (cPlatform V3) issued from IMAGINE and CRESCENDO. The adopted approach combined usage of Enterprise Applications virtualization and infrastructure (machines and networks) virtualization as described on Figure 7.

cPlatform was designed for delivering cross organization collaborative services for PLM, aggregating standardized enterprise application components such as Enterprise Horizontal Portal based on Portal standards, Enterprise Service Bus based on Web standards, Enterprise Workflow Systems based on Enterprise Workflow standards. It was architected as a hub where the different applications involved in the collaboration can be plugged when starting a collaboration and unplugged, being interfaced with the Enterprise portal through portlets for secured roles-based access managed by the portal, and with the Enterprise Service Bus for middleware interconnection when required. At the front end, cPlatform reflects the whole dynamic networked organization working around a product, so a DMN. However, the actual location of the applications is hidden, so it is not possible to know if the application is hosted by one of the partners or by a cloud platform provider. It means that the Application of Reference Components, as interconnected by standardized interface, can be transparently replaced by any other compliant component as soon as validated (can play the expected role in the Business Collaboration) and verified (can interoperate with other applications using the Application protocol) by mean of the SIP Platform.

The interoperability must be addressed on all the layers, including the technical one with software systems running on machines distributed on and connected through networks of networks, themselves distributed on the information systems of several enterprises, as part of the front office, the back-office or the middleware. For security reasons, the access to applications and private networks is restricted. For testing interoperability on this layer, the idea is to simulate the relevant part of the infrastructure by means of the Network Virtualization System. With such a solution, actual structure in terms of machines and networks of networks can be simulated, reflecting the used security policies in order to prove they are not preventing interoperability. Many machines and their context can be simulated with a single physical machine, or eventually a set of machines in a single cluster which is managed transparently by the Network Virtualization System. Finally, all the people involved in the PLM standard assessment can 
access it through experimentation reflecting actual distribution: the cPlatform extended with SIP services is deployed on the Cloud. This was not the case for the first version of the cPlatform. Virtualization was introduced by SIP as an important enabler for simulation of an actual multi-enterprise collaborative system with limited resources (in particular the number of physical servers) . Virtualization however allows keeping the main functionalities, distributed software components and multi-layered protocols on demand. Indeed, testing on a replication of an actual system is too expensive and is not necessarily a guarantee of success because minor differences between the actual system and the replicated system may be at the origin of non -interoperability. The assessed Cloud virtualization technologies to be used for covering PLM interoperability testing needs were categorized according to a specifically defined classification. At the ICT layer, performed assessment concerned relevant solutions for producing a multitenant network of virtual networks. As a result, a network virtualization solution (Proxmox) was chosen, supporting various types of hypervisors and virtual machine containers.

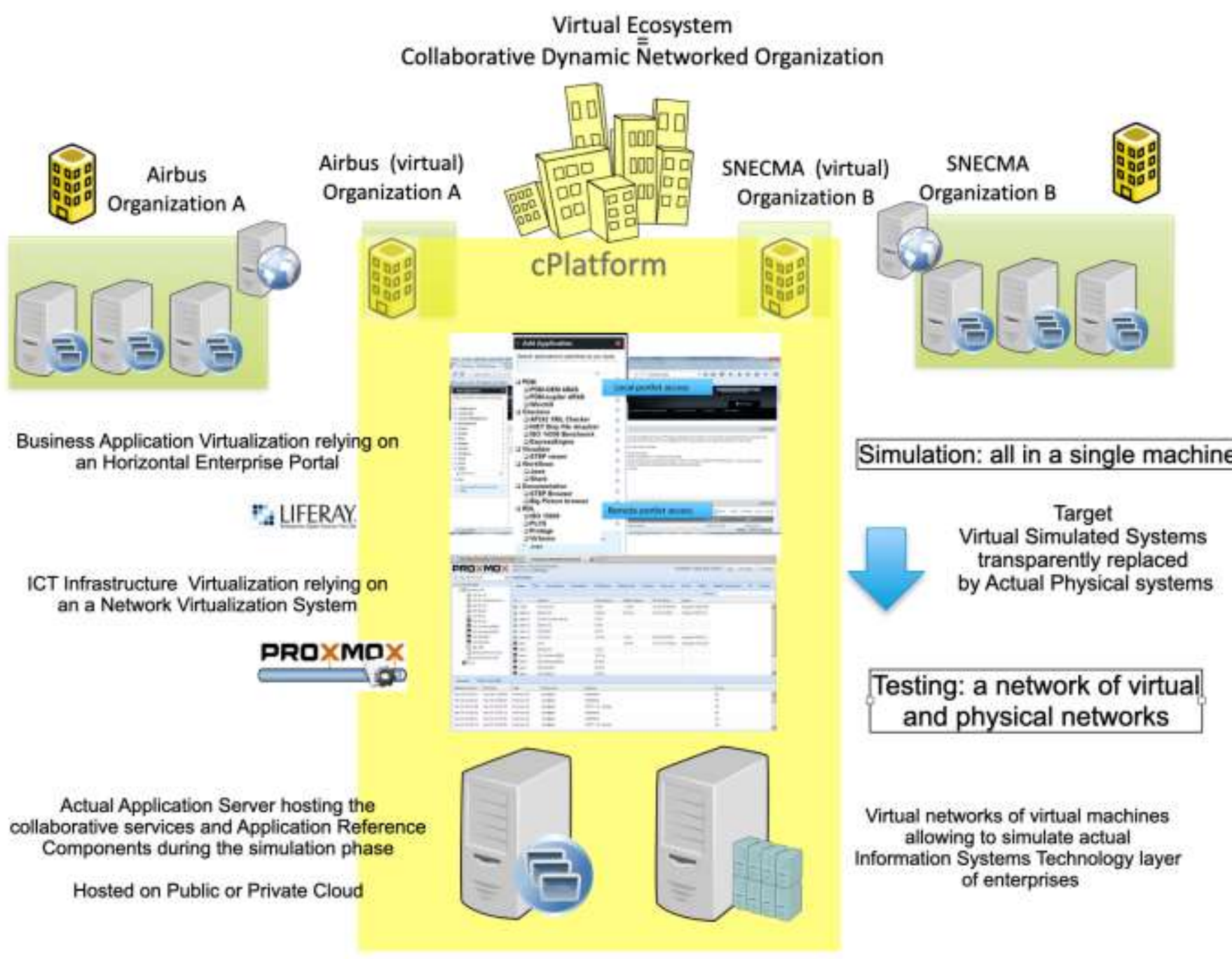

Figure 7: Combination of ICT infrastructure and Application virtualization

At application layer, the relevant identified categories of enterprise solutions are enterprise horizontal portal (Liferay), workflow systems and Enterprise Service Bus.

In terms of virtualization of applications distributed in networked organizations, the portal is the most important solution component, fitting perfectly well with infrastructure network virtualization solutions. The considered criteria for assessing the categories of solutions were simplicity, openness, robustness and reusability as parametric building blocks of the DSF. It means it should be easy to deploy and aggregate them for building and reconfiguring on demand complex heterogeneous simulation and PLM testing infrastructures. A model based automating creation of components relying on such building blocks was defined as illustrated by Figure 8. It will be assessed in operation and the results will be the subject of future research publications. Such Model Based System Engineering usually applies to manufactured products. We demonstrated it can be applied to composite 
organizations too, using standard libraries of preinstalled machines (appliances) and associated parametric models formalized as ArchiMate blueprint models. In the figure, we used LEGOs analogy, with first creation and assembly by hand, i.e. non-automated process for infrastructure set up. In a second stage, provisioning, assembly and deployment are automated using parametric DMN blueprint templates, associated with previously defined and validated appliances. Figure 8 shows the model of the collaboration described in Figure 4. Used models repositories concern DMN blueprint templates, test data sets based on used exchange technologies and processes

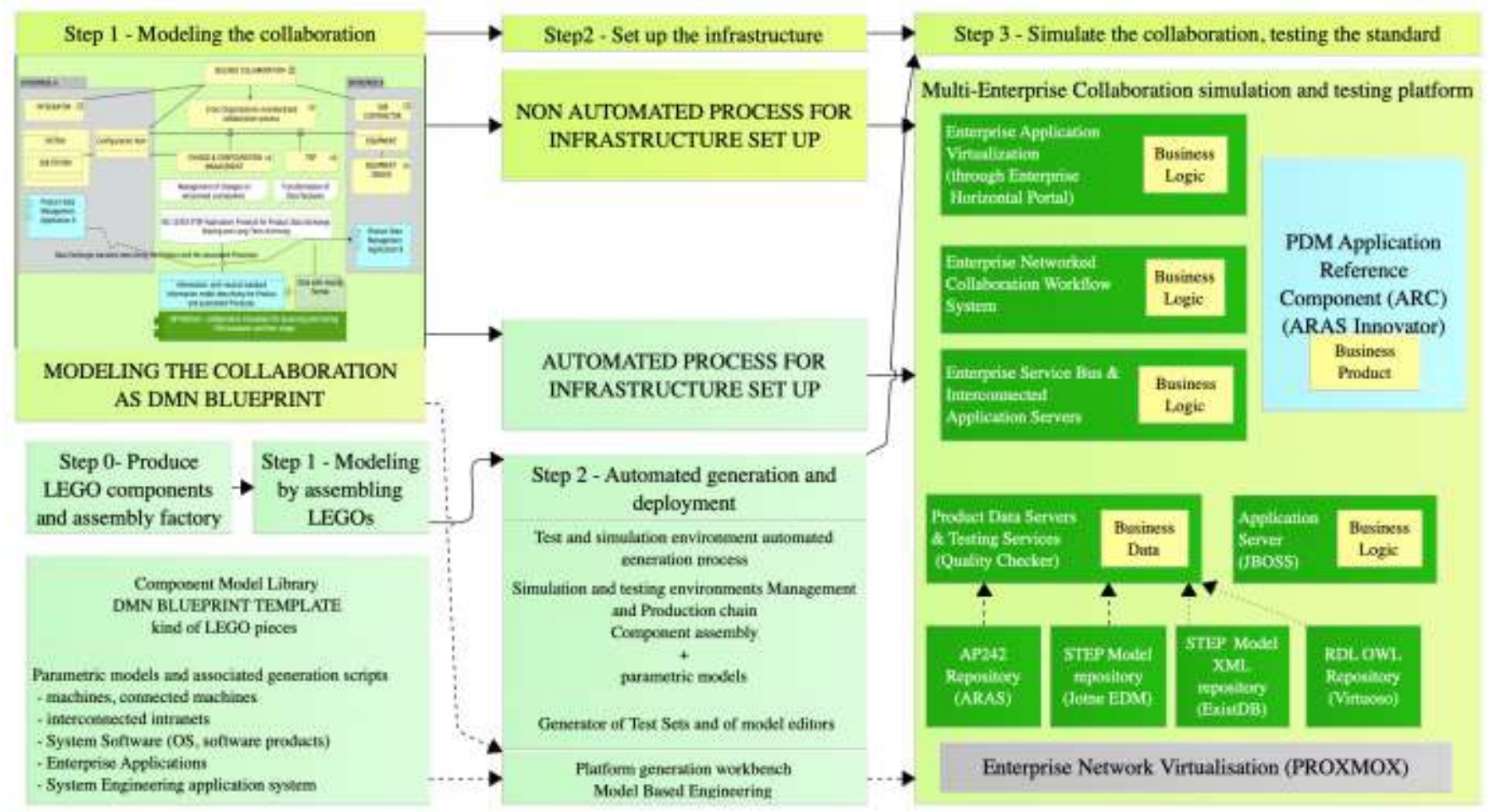

Figure 8: Cloud Virtualization assessment for the Interoperability Test bed

This automation is currently being validated and will be the purpose of the next FIF evolutions, with an updated and extended cPlatform. It illustrates and demonstrates that the FIF can easily be extended and adapted to the changing business (usage of new protocols) and technological (Cloud) context. The contribution the SoA is reflected in table 3.

\begin{tabular}{|c|c|l|}
\hline FIF related Paper & Year & Contribution to the FIF \\
\hline$[9]$ & 2016 & $\begin{array}{l}\text { Multi-level approach based on the use of standards in a DMN. The proposed approach is highlighted by a real manufacturing } \\
\text { case study relying on [ISA95] for MES to ERP communication. }\end{array}$ \\
\hline$[10]$ & 2016 & This paper presents the DMN Software Factory \\
\hline
\end{tabular}

Table 3: FIF last iteration related publications

At the same time, future evolutions have been already considered to deal with interoperability challenges for Internet of Things technologies used in combination with Virtual Manufacturing or CPS.

\section{The Future Evolutions For Virtual MANufacturing AND Cyber PhysicAl Systems}

The previous sections explained the past and current evolutions of the FIF for a continuous adaptation to the business and technological context. This section explores next evolutions to support PLM of the future needs..

New use cases for the FIF have been initiated concerning assessment of emerging standard frameworks for Virtual Manufacturing taking advantage of Internet emerging technologies. It should support usage of digital twins, smart manufacturing, or PLM for circular economy. For mature communities in terms of interoperability, some consensus exists at national initiative levels around usage of models of reference [RAMI] [NIST reference model] or some other countries' initiatives. The FIF initially addressed interoperability of Enterprise Applications, and not of products or production systems connected to the enterprise information systems. Why can the FIF be easily extended, taking advantage of what was already developed and support the assessment of the joint usage of standard components of the emerging architectures or reference? 
First the last ArchiMate version (3.1) was assessed in terms of support of new use cases with description of manufacturing capabilities and IoT technology. The new physical layer comes with the modeling constructs "Equipment", "Facility", "Distribution Network" and "Material". Factories can be modeled with captors or actuators connected to the Cloud; reflecting the layers of Architecture of Reference of the Manufacturing domain, such as [PERA] or [RAMI], as the ArchiMate view on Figure 9 demonstrates it.

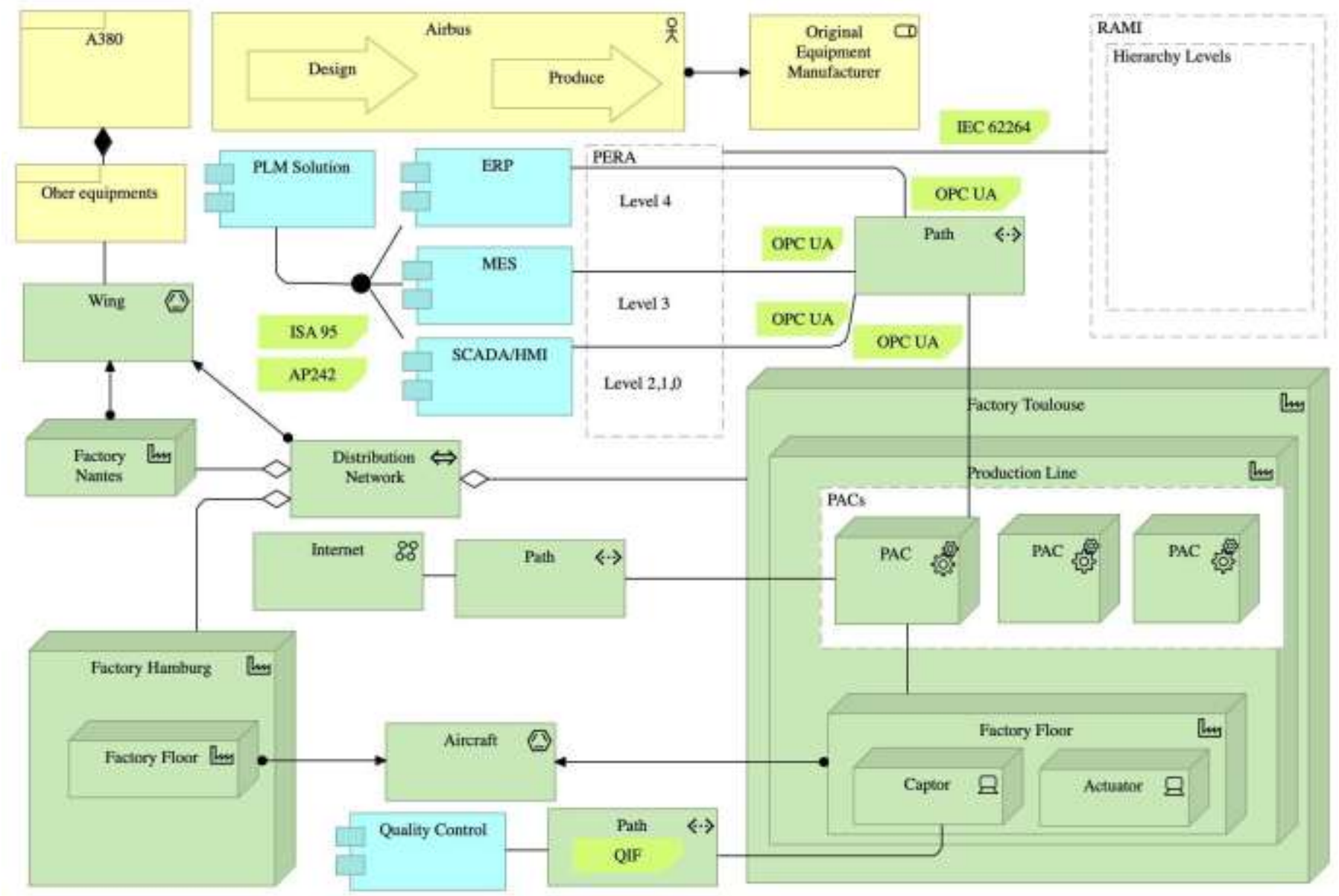

Figure 9: Manufacturing and IIoT standards supported by ArchiMate 3.1

Second new kinds of technologies, such as deep learning, big data or blockchain solutions, and the maturity of the related open standards, can be easily assessed, with updates of the strategic roadmap and ArchiMate blueprint models.

Third experimental testing platforms integrating and simulating new building blocks can be easily built and are highly facilitated by emergence of standards with available open-source solutions for captors, actuators and associated protocols. E.g. [OPC UA], an identified building block of The Industrie 4.0, comes with many freely available open source implementations.

Fourth the different standards can be assessed standalone or combined together with continuous integration using the FIF. We can rely on already performed previous assessments and testing platform components. E.g. [ISA 95] was already assessed with the FIF in combination with other standards, in IMAGINE and SIP projects. It is a higher level standard describing manufacturing operations management domain and its activities between the plants and the enterprise, and formed the basis of Manufacturing Execution Systems (MES) functions.

Finally the assessment and testing approach defined for the FIF can be applied for assessing IoT or cyber-physical systems related standards: 1) the concept of test bed as defined in the FIF was derived from one approach already used in aeronautic for aircrafts, for which a version 0 (Iron bird) is created, having the same role and adopting the same principle than the test bed. Consequently, it can also be applied to a combination of software and cyber-physical systems. 2) the emerging trends related to makers and open source for hardware and IoT should enable the creation of a low price and evolutionary platform for assessment and testing of IoT standards. Assessing such feasibility was initiated considering open source connected drones or autonomous connected robots. Here again, open source is used, but for hardware as well as for software. But what is quite more interesting with these products for makers is that they can be used for experimenting the whole life cycle of a product, from design to maintenance, and considering eventually their evolution. The components constituting the bills of material are available at a low price and some of them can be printed using a 3D printer. Definitions, models or assembly process are also made available. Associated embedded software (e.g. for autonomous robots) and applications allowing to connect them to the Internet (e.g. for piloting them) are also 
available in open source, sometimes free, and relying on some emerging standards. It means that it should be possible to build richer test cases for PLM interoperability, including virtual manufacturing or cyber physical systems such as connected drones or autonomous robots. It should also allow extending the test bed and the cPlatform with complementary collaborative and testing capabilities for the new emerging trends impacting the PLM, such as digital twins, digital threads, or circular economy. The open source for CPS combined with emerging IoT standards becomes a new enabler for interoperability, as it is already the case for software. The FIF being built on top of these enablers, extending the FIF and making it evolve to cover these new use cases should not be a problem. It is one envisaged evolution of the FIF which should open many perspectives.

In parallel to the preparation of next evolution, the FIF community is still contributing to the state of the art as reflected by table 4.

\begin{tabular}{|l|l|l|}
\hline FIF related paper & \multicolumn{1}{|c|}{ Year } & Contribution to the FIF \\
\hline [23] & 2019 & $\begin{array}{l}\text { This paper addresses some limitation of the EH4I when having to deal with multi-scale models to be used for complex } \\
\text { systems of systems. It proposes an innovative approach for extending the FIF, relying on modeling over UML2/SysML } \\
\text { and introduces mereotopology. Usage is illustrated with a comprehensive use case combining Manufacturing 4.0 and PLM } \\
\text { of the future. }\end{array}$ \\
\hline
\end{tabular}

Table 4: FIF related last publications

As a conclusion, FIF evolvability is demonstrated for the future business cases related to Virtual Manufacturing, CPS and PLM of the future.

\section{DISCUSSION CONCERNING EVOLUTIONARY SYSTEMS AND INTEROPERABILITY}

In this section, we discuss the required evolutionary nature of systems for interoperability. We then show how it makes the FIF accurate and original, compared to other interoperability frameworks, for preparing and building continuous operational PLM interoperability.

[24] and [25] are discussing the different non-functional qualities of a system, called the "ilities", in order to contribute toward a prescriptive "Theory of Ilities". After pointing out some semantic challenges related to "ilities" related to ambiguity in terms, polysemy, synonymy, one " ility" considered at a time, particular sets of related "ilities" are proposed, based on a study on how the "ilities" are related to one another, with the re-examination of their definitions (cf. table 5)

\begin{tabular}{|l|l|}
\hline Agility & to change in a timely fashion \\
\hline Changeability & to alter its operations or form, and consequently possibly its function, at an acceptable level of resources \\
\hline Evolvability & to be designed for inheriting and changing across generations (over time) \\
\hline Extensibility & to accommodate new features after design \\
\hline Flexibility & to be changed by a system-external change agent with intent \\
\hline Interoperability & to effectively interact with other systems \\
\hline Reconfigurability & to change its component arrangement and links reversibly \\
\hline
\end{tabular}

Table 5: "ilities" definitions

These definitions were produced in order to be able to group the "ilities" as a "means-ends" hierarchy, i.e. one that represents the relationships between ilities in terms of using one ility as a "means" for accomplishing another ility ("ends").

According to the performed analysis, it seems that some "ilities" become more important over time and that some are still in a nascent state and may not yet have developed their own set of supporting "ilities". It includes sustainability, resilience, Evolvability but also surprisingly interoperability. It is surprising because such an "ility" has been studied for years by several communities, and because [2] identified interoperability enablers and strong relationships with other "ilities".

Within the frame of this paper, we agree with the [24] and [25] proposed definitions. We also consider that evolvability is a high level "ility". But the return on experience leads to a different hierarchy of "ilities": Evolvability implies mainly a system to be changeable, extensible, agile, flexible and reconfigurable. It is in fact the foundation of PLM and Product Data Management, for which the definition of a product is something continuously evolving and managed through the change and configuration management processes. In addition, if a supporting system such as the cPlatform is not evolutionary, it will not be usable to maintain the continuous PLM interoperability for complex DMN systems. The other "ilities" can also impact interoperability, but to a lower level. Performing the exercise (cf. table 6), it also appears that the FIF approach is robust as it can maintain the provided 
value despite external changes (context, needs) and internal changes (continuous extension and reconfiguration of the FIF platforms).

SIP highly contributed to the changeability and agility of the FIF, by supporting reduction of resources (time and cost) for the evolution of PLM hub and of a test bed. The principle of using a set of configured standards highly contributes to the configurability and of the flexibility. They also contribute to the changeability and agility when used technical solutions are standard compliant and when the standards are suitable for interchangeable components.

The return on experience of the many successive projects and the open perspectives for FIF evolution for dealing with the PLM of the future proved the evolutionary nature of the FIF, which make it suitable for establishing continuous interoperability for long term.

\begin{tabular}{|l|l|l|l|}
\hline Target "ility" & \multicolumn{2}{|l|}{ Supporting "ilities" } & Evidence that FIF has such abilities \\
\hline Interoperability & Evolvability & $\begin{array}{l}\text { FIF was demonstrated as changeable, extensible, reconfigurable, agile and flexible. It was able to support } \\
\text { several generations of technologies and practices, including all those illustrated in this article. The current } \\
\text { studies show that it should also be the case for virtual manufacturing (initiated) and CPS (assessed). }\end{array}$ \\
\hline & Changeability & $\begin{array}{l}\text { The FIF can be changed fast, due to the adopted architectural patterns and usage of commodities on the } \\
\text { WEB, i.e. blocks that can be considered as Lego with standardized interfaces }\end{array}$ \\
\hline & Extensibility & $\begin{array}{l}\text { The FIF can be extended in order to support new functional areas (e.g. virtual manufacturing) and with new } \\
\text { enablers such as virtualization and cloud technologies. Extension of ArchiMate makes it useable for } \\
\text { manufacturing and virtual manufacturing. }\end{array}$ \\
\hline & $\begin{array}{l}\text { Reconfigurabil } \\
\text { ity }\end{array}$ & $\begin{array}{l}\text { The FIF have been continuously reconfigured from ATHENA to SIP and after, as described in sections II } \\
\text { \& III, with evolution of versions of the implementation components and of the used open standards. }\end{array}$ \\
\hline & Agility & $\begin{array}{l}\text { The FIF has been changed fast, due to the adopted architectural patterns and usage of commodities on the } \\
\text { Web, i.e. blocks that can be considered as Lego with standardized interfaces }\end{array}$ \\
\hline & Flexibility & The FIF has been changed by a system external change agent with intent, by design \\
\hline
\end{tabular}

Table 6: Evidence that FIF is evolutionary

Compared to other interoperability frameworks proposed by the SoA and the SoP, the FIF brings something new. It should be mentioned that such a comparison is difficult due to the nature of the FIF. First it is not yet another interoperability framework, but an approach aiming at federating usage of existing and relevant ones. The frameworks to consider will depend on the purpose and on the nature of the systems which must interoperate for a given business ecosystem. Another important point is that the FIF aims at preparing and building operational interoperability, not in theory and in a purely academic context, but relying on a dynamic between research, standardization, and operational projects, taking the best of the state of the art and of the state of the practice. The return of experience from the research projects described in this paper demonstrates that such separation between research and operation is one very important interoperability brake [2].

On the reverse, outputs from research on interoperability are rarely used, because other important non-functional properties of an operational system (e.g., security, dynamic reconfiguration) are most of the time not considered by the Interoperability research community. A last reason why comparison is difficult comes from the fact interoperability is something qualitative, and nonquantitative and cannot consequently be measured. Indeed, even if we can define qualitative key indicators, we can't say a system is two times more interoperable than another. It is interoperable or it is not. However, it is possible to quantify what the FIF brings with some figures used for the successive research and operational projects which contributed to develop and to assess the FIF. First, the FIF approach allowed successive projects to change the ratio of effort between the setup of infrastructure and functional assessment from $80 \% / 20 \%$ to $20 \% / 80 \%$.

The main enabler for this was the adoption of a model-based approach based on the [MDA]). Second, relying on open and mature international standards allows setting-up strategic governance. It was demonstrated by the setup of the ASD SSG [26], which was motivated by the presentation of the results of [2] and its usage in the S.E.I.N.E. project [27]. Finally, FIF considers unique features not supported by other interoperability frameworks: the concept of extended hyper model for interoperability [23], the concept of Dynamic Manufacturing Network which was developed within the context of the IMAGINE project [24] and finally the combination of usage of semantic graph (ontology) with mereotopology (i.e. the study of the whole and parts combined with topology), when considering dynamic manufacturing networks as complex SoS with multiple granularities.

Many existing architecture frameworks dealing with interoperability have been considered, not suited for enterprise application interoperability, but bringing useful concepts and principles. In particular, the System Of Systems Interoperability framework brought the operational interoperability concept, but it is not accurate for Enterprise Application Interoperability. ETSI interoperability framework [28] is coming with the concept of testbed but it is applied only to ICT infrastructure. Many interoperability frameworks related to data [STEP], semantic web [OWL], software system [CORBA], processes, services, 
messages, or events were considered, relying on a given paradigm and consequently bringing some limitations when having to cover the whole spectrum of needs for Enterprise Interoperability. The FIF aims at combining consistent subsets of these frameworks which will support governing and building the targeted continuous interoperability. The table 7 reflects some of the main frameworks which have been assessed and considered, and that could be compared to the FIF.

\begin{tabular}{|l|c|c|c|c|c|c|c|}
\hline & FIF & SOSI & ETSI & CORBA with OMA & STEP Framework & W3C Semantic Wcb & AIF \\
\hline Operational Interoperability & Y & Y & N & N & N & N & N \\
\hline Test orientation & Y & N & Y & N & N & N & N \\
\hline Hypermodel concept & Y & N & N & N & N & N & N \\
\hline Composite collaborative modelling & Y & N & N & N & N & N & N \\
\hline Considering open source as an enabler & Y & N & N & N & N & N & N \\
\hline Considering business open standards & Y & N & N & N & Y & N & N \\
\hline Considering Interoperability brakes & Y & N & N & N & N & N & N \\
\hline DMN & Y & N & N & N & N & N & N \\
\hline Evolutionary & Y & N & N & N & N & Y & N \\
\hline
\end{tabular}

Table 7: Interoperability Frameworks comparison

For highlighting the FIF the scientific contribution in terms of interoperability, table 8 provides recent papers in the domain identified through a SoA performed specifically for this paper. Some Interoperability Framework such as LISI (Levels of Information Systems Interoperability), NATO's LCIM (Levels of Conceptual Interoperability Model), LCI (Layer of Coalition Interoperability), Interoperability Maturity Models such as National eHealth Transition Authority Interoperability Model (NEHTA 2007) are not mentioned, as their description, contribution and positioning with the FIF were already provided by [2] and [25].

\begin{tabular}{|c|c|c|c|}
\hline Paper title & Main result & What is missing & FIF contribution \\
\hline $\begin{array}{l}\text { Towards a Classification } \\
\text { Framework for Interoperability of } \\
\text { Enterprise Applications [29] }\end{array}$ & $\begin{array}{l}\text { Classifications based on a } \\
\text { set of frameworks for } \\
\text { defining the expected } \\
\text { interoperability }\end{array}$ & $\begin{array}{l}\text { Characterization of the ideal } \\
\text { PLM system, with } \\
\text { associated enablers and } \\
\text { breakthroughs }\end{array}$ & $\begin{array}{l}\text { Application of the proposed classification, with } \\
\text { identification of a set of enablers and } \\
\text { breakthroughs to be addressed for preparing } \\
\text { and building an operational interoperability }\end{array}$ \\
\hline $\begin{array}{l}\text { GRAI integrated methodology } \\
\text { and its mapping onto generic } \\
\text { enterprise reference } \\
\text { architecture and methodology } \\
\text { [30] }\end{array}$ & $\begin{array}{l}\text { Integrated Enterprise } \\
\text { modelling methodology } \\
\text { including decision model }\end{array}$ & $\begin{array}{l}\text { Stronger focus on Business } \\
\text { Production and Motivation. } \\
\text { Difficult to adapt to emerging } \\
\text { Virtual Manufacturing }\end{array}$ & $\begin{array}{l}\text { Simultaneous coverage of Business, Information } \\
\text { System, and ICT layers, all along the different } \\
\text { phases of Product Life Cycle. FIF designed for } \\
\text { being evolutionary }\end{array}$ \\
\hline $\begin{array}{l}\text { Model-based approaches for } \\
\text { interoperability of the next } \\
\text { generation enterprise } \\
\text { information systems - state of } \\
\text { the art and future challenges } \\
\text { [31] }\end{array}$ & $\begin{array}{l}\text { New challenges for the } \\
\text { interoperability of } \\
\text { Enterprise Information } \\
\text { System related to } \\
\text { modeling, with } \\
\text { identification of barriers }\end{array}$ & $\begin{array}{l}\text { Some of the identified } \\
\text { barriers for next generation } \\
\text { Enterprise Integration } \\
\text { Systems (EIS) are very old } \\
\text { barriers. The impact of high } \\
\text { pace of change for } \\
\text { technologies, being ICT or } \\
\text { modeling, and requiring } \\
\text { evolutionary framework, is } \\
\text { not well stated, as } \\
\text { importance of testing } \\
\text { capabilities for assessing } \\
\text { and maintaining } \\
\text { interoperability }\end{array}$ & $\begin{array}{l}\text { FIF established the link between Enterprise } \\
\text { Modelling used for governance and continuous } \\
\text { evolution of the Enterprise Information System, } \\
\text { relying on an evolutionary framework that can } \\
\text { be reconfigured and extended in order to follow } \\
\text { evolving needs and context of the enterprises. } \\
\text { Testability (SIP) and configurability have been } \\
\text { addressed [11], as well as reverse modelling } \\
\text { and semantic preservation through usage of } \\
\text { hyper-models. Most of the addressed } \\
\text { challenges were or have been addressed during } \\
\text { the FIF development. }\end{array}$ \\
\hline
\end{tabular}


ICMS: A cloud-based manufacturing system [32] and Assessment of interoperability in cloud manufacturing. Robotics and ComputerIntegrated Manufacturing [33]
An Interoperability framework relying on STEP NC and MT connect, and Viability of Cloud Manufacturing when having to ensure interoperability
Interoperability itself is not assessed and the scope is restricted to manufacturing (production)
The interoperability is assessed considering the whole supply chain and the whole life cycle of the manufactured products, and not restricted to 2 standards

Table 8: Positioning within the SoA

Considering the SoAs of the referenced SIP papers in each section, and the most recent one we performed specifically for this paper an reflected in in table 8, we didn't identified any interoperability framework pointing out and addressing the required evolutionary nature of an interoperability framework aiming at preparing, building and maintaining PLM continuous operational interoperability at an acceptable price for a long term duration, and being able to aggregate and federate several approaches.

Finally, it should be considered that as stated by [34], the research community in the interoperability and integration testing of IoT systems, which impact virtual manufacturing and CPS, is rather heterogeneous, due to the novelty of the area. Some maturity is expected before obtaining mature enough standards. We demonstrated in this paper that the FIF can contribute for building and assessing such maturity.

\section{CONCLUSION}

The Federated Interoperability Framework (FIF) has been developed continuously for Aeronautic Space and Defence over the last years in order to prepare and to build the continuous Product Lifecycle Management (PLM) interoperability at an acceptable cost. This paper demonstrated the evolutionary nature of the FIF. After describing the FIF, its genesis and the associated experimental and testing platform which encompass evolvability in their requirements, the different evolutions over a period of twenty years were described, from the genesis (section II) to the last evolution (section III), as well as the planned evolutions for supporting emerging Virtual Manufacturing and Cyber-Physical-Systems which will impact the PLM of the future.

Cloud and Portal virtualization technologies were integrated in the FIF in order to shorten the time-to-usage for PLM standards within a Dynamic Manufacturing Network (DMN). Similarly, FIF is being extended in order to identify and address new interoperability challenges and scientific gaps related to Virtual Manufacturing or CPS, with the usage of emerging Internet technologies. It provides a way for shorting the time of using emerging standards for Manufacturing 4.0 or digital twins. Important identified scientific gap to address in the future is related to the need to consider complex SoS for which the systemic paradigm is less and less accurate when considering multi-scale virtualized systems. Other perspectives are the integration of emerging technologies such as IIoT, blockchain and deep learning as building blocks of the cPlatform with the assessment of related standards to be part of the previously identified relevant sets of consistent open standards.

\section{REFERENCES}

1. “About PLM", CIMDATA, http://www.cimdata.org/

2. "Interoperability of technical enterprise applications", N. Figay. Other [cs.OH]. Université Claude Bernard - Lyon I, 2009. English. NNT : 2009LYO10242

3. SystemX Standard Interoperability PLM project web site at http://www.irt-systemx.fr/systemx-lance-le-projet-sip-standardsinteroperabilite-plm/Data

4. Figay N., Ghodous P. (2008) Collaborative Product Development: EADS Pilot Based on ATHENA. In: Mertins K., Ruggaber R., Popplewell K., Xu X. (eds) Enterprise Interoperability III. Springer, London. https://doi.org/10.1007/978-1-84800-221-0_33

5. ATHENA Interoperability Framework available at https://sintef-9012.github.io/athena-interoperability-framework/

6. N. Figay and P. Ghodous, "FLOSS as Enterprise Application Interoperability Enabler," 2009 Fifth International Conference on Signal Image Technology and Internet Based Systems, Marrakesh, 2009, pp. 435-442, doi: 10.1109/SITIS.2009.74.

7. D.Chen and al, A Maturity Model for Enterprise Interoperability, 2009. In: « On the Move to Meaningful Internet Systems: OTM 2009 Workshops. OTM 2009. Lecture Notes in Computer Science, vol 5872. Springer, Berlin, Heidelberg. https://doi.org/10.1007/978-3-642-05290-3_32

8. IMAGINE: "Innovative end-to-end Management of Dynamic Manufacturing Networks», http://www.imaginefuturefactory.eu project wet site at http://www.eads-iw.net/web/imagine

9. D. Tchoffa, N. Figay, P. Ghodous, E. Exposito, Lyes Kermad, Thomas Vosgien, A. El Mhamedi, Digital factory system for dynamic manufacturing network supporting networked collaborative product development, Data \& Knowledge Engineering,Volume 105,2016,Pages 130-154,ISSN 0169-023X, https://doi.org/10.1016/j.datak.2016.02.004.http://www.sciencedirect.com/science/article/pii/S0169023X16300040

10. N.Figay, D.Tchoffa, P.Ghodous, E.Exposito, A.E.Mahmedi, Dynamic manufacturing network - from flat semantic graphs to composite model, February 2019, International Journal of Production Research 57(20):1-10, DOI: 10.1080/00207543.2019.1570375

11. Moones E. et al. (2015) Towards an Extended Interoperability Systemic Approach for Dynamic Manufacturing Networks: Role and Assessment of PLMStandards. In: Boulanger F., Krob D., Morel G., Roussel JC. (eds) Complex Systems Design \& Management. Springer, Cham. https://doi.org/10.1007/978-3-319-11617-4_5

12. Figay N., Tchoffa D., Ghodous P., Exposito E., El Mhamedi A. (2014) Dynamic Manufacturing Network, PLM Hub and Business Standards Testbed. In: Mertins K., Bénaben F., Poler R., Bourrières JP. (eds) Enterprise Interoperability VI. Proceedings of the I-ESA Conferences, vol 7. Springer, Cham. https://doi.org/10.1007/978-3-319-04948-9_38 
13. N. Figay and P. Ghodous, "Extended Hypermodel for Interoperability within the Virtual Enterprise," 2009 Fifth International Conference on Signal Image Technology and Internet Based Systems, Marrakesh, 2009, pp. 393-400, doi: 10.1109/SITIS.2009.68.

14. Jardim-Goncalves, N.Figay, A. Steiger-Garcao. Enabling interoperability of STEP Application Protocols at meta-data and knowledge level», International Journal of Technology Management,2006,, Volume 36 Issue 4, p402-421

15. Nicolas Figay, Parisa Ghodous, Malik Khalfallah, Mahmoud Barhamgi, Interoperability framework for dynamic manufacturing networks, Computers in $\begin{array}{llll}\text { Industry,Volume } & 63, & \text { Issue } & 8,2012, \text { Pages }\end{array}$ 3615,https://doi.org/10.1016/j.compind.2012.08.008.(http://www.sciencedirect.com/science/article/pii/S0166361512001273)

16. « Pragmatic PLM Process Interoperability for Aeronautic, Space and Defence DMN », N. Figay, P. Ghodous, C. Ferreira Da Silva, S. Ghafour, Journal of Aerospace Operations, vol. 3, no. 3,4, pp. 147-166, 2015

17. E.Moones, L.Kermad, T.Vosgien, E.M.Dafaoui, A.E.Mahmedi, N.Figay, PLM standards modeling for enterprise interoperability: A manufacturing case study between ERP and MES information exchange business scenario based on ISA 95 standard, (2015) IWEI 2015. Lecture Notes in Business Information Processing, vol 213 p157. Springer, Berlin, Heidelberg

18. N. Ivezic, B. Kulvatunyou, A.. Jones, A manufacturing B2B interoperability testbed, ICEC ' 03 Proceedings of the 5th international conference on Electronic commerce ,Pages 195-204

19. ETSI, «Interoperability Best Practices Edition 2», (2008) ETSI, http://www.etsi.org/about/how-we-work/testing-andinteroperability

20. Figay N., da Silva C.F., Ghodous P., Jardim-Goncalves R. (2015) Resolving Interoperability in Concurrent Engineering. In: Stjepandić J., Wognum N., J.C. Verhagen W. (eds) Concurrent Engineering in the 21st Century. Springer, Cham. https://doi.org/10.1007/978-3-319-13776-6_6

21. Nicolas Figay, Parisa Ghodous, Malik Khalfallah, Mahmoud Barhamgi, Interoperability framework for dynamic manufacturing networks, Computers in Industry,Volume $63, \quad$ Issue $\quad 8,2012$,Pages 3615,https://doi.org/10.1016/j.compind.2012.08.008.(http://www.sciencedirect.com/science/article/pii/S0166361512001273)

22. V. Rozsa, M. Denisczwicz, M. Dutra, P. Ghodous, C.Ferreira da Silva, N.Moayeri, F. Biennier, N. Figay, « An Application Domain-Based Taxonomy for IoT Sensors, Series "Advances in Transdisciplinary Engineering", Volume 4: Transdisciplinary Engineering: Crossing Boundaries, ISPE TE 2016: 249-258

23. N.Figay, D.Tchoffa, P.Ghodous, E.Exposito, A.E.Mahmedi, Dynamic manufacturing network - from flat semantic graphs to composite model, February 2019, International Journal of Production Research 57(20):1-10, DOI: 10.1080/00207543.2019.1570375

24. O.de Weck, A. M.Ross, and D. H. Rhodes, Investigating Relationships and Semantic Sets amongst System Lifecycle Properties (Ilities), Third International Engineering Systems Symposium, CESUN 2012, Delft University of Technology, 18-20 June 2012

25. A. M .Ross, A Prescriptive Semantic Basis for System Lifecycle Properties, seari.mit.edu, April 2014

26. ASD Strategic Standardization Group web site at http://www.asd-ssg/org

27. SEINE Project web site at http://seine.afnet.fr/

28. ETSI, «Interoperability Best Practices Edition 2», (2008) ETSI, http://www.etsi.org/about/how-we-work/testing-andinteroperability

29. H. Panetto, "Towards a Classification Framework for Interoperability of Enterprise Applications", International Journal of Computer Integrated Manufacturing, Taylor \& Francis, 2007, 20 (8), pp.727-740. <10.1080/09511920600996419>

30. "GRAI integrated methodology and its mapping onto generic enterprise reference architecture and methodology", Chen, David, Bruno Vallespir, and Guy Doumeingts. Computers in industry 33.2 (1997): 387-394.

31. Model-based approaches for interoperability of next generation enterprise information systems: state of the art and future challeng es"Gregory Zacharewicz, Saikou Y. Diallo, Yves Ducq, Carlos Agostinho, Ricardo Jardim-Gonçalves, Hassan Bazoun, Zhongjie Wang, Guy Doumeingts:. Inf. Syst. E-Business Management 15(2): 229-256 (2017)

32. X. V. Wang, X. W. Xu, Cloud Manufacturing, in: W. Li, J. Mehnen (Eds.), ICMS: A Cloud-Based Manufacturing System, Springer Series in Advanced Manufacturing, Springer London, London, 2013, Ch. 1, pp. 1-22. doi:10.1007/978-1-4471-4935-4.

33. Mourad, M., Nassehi, A., Schaefer, D., \& Newman, S. T. (2020). Assessment of interoperability in cloud manufacturing. Robotics and Computer-Integrated Manufacturing, 61, [101832]. https://doi.org/10.1016/j.rcim.2019.101832

34. Bures M. et al. (2020) Interoperability and Integration Testing Methods for IoT Systems: A Systematic Mapping Study. In: de Boer F., Cerone A. (eds) Software Engineering and Formal Methods. SEFM 2020. Lecture Notes in Computer Science, vol 12310. Springer, Cham. https://doi.org/10.1007/978-3030-58768-0_6 